\title{
On a generalization of the Mukai conjecture for Fano fourfolds
}

\author{
Kento Fujita
}

November 10, 2018

\begin{abstract}
Let $X$ be a complex $n$-dimensional Fano manifold. Let $s(X)$ be the sum of $l(R)-1$ for all the extremal rays $R$ of $X$, the edges of the cone $\mathrm{NE}(X)$ of curves of $X$, where $l(R)$ denotes the minimum of $\left(-K_{X} \cdot C\right)$ for all rational curves $C$ whose classes $[C]$ belong to $R$. We show that $s(X) \leq n$ if $n \leq 4$. And for $n \leq 4$, we completely classify the case the equality holds. This is a refinement of the Mukai conjecture on Fano fourfolds.
\end{abstract}

\section{Introduction}

Let $X$ be an arbitrary $n$-dimensional Fano manifold with the Picard number $\rho_{X}$. In 1988, Mukai Muk88 made the following conjecture.

Conjecture 1.1. One has

$$
\rho_{X}\left(r_{X}-1\right) \leq n,
$$

and the equality holds if and only if $X \simeq\left(\mathbb{P}^{r_{X}-1}\right)^{\rho_{X}}$, where

$$
r_{X}:=\max \left\{m \in \mathbb{Z}_{>0} \mid-K_{X} \sim m L \text { for some Cartier divisor } L\right\} .
$$

There are several approaches and refinements of Conjecture 1.1. See for example [ACO04, BCDD03, Cas06, NO10, Wiś90]. Nowadays, the following conjecture due to Tsukioka Tsu12] (cf. Tsu10c]) is the most generalized version of Conjecture 1.1.

Conjecture 1.2. One has

$$
\rho_{X}\left(l_{X}-1\right) \leq n,
$$

and the equality holds if and only if $X \simeq\left(\mathbb{P}^{l_{X}-1}\right)^{\rho_{X}}$, where $l_{X}$ denotes the minimum of the length $l(R)$ of all the extremal rays $R$ of $X$, and

$$
l(R):=\min \left\{\left(-K_{X} \cdot C\right) \mid C \subset X \text { is a rational curve with }[C] \in R\right\} .
$$


We think that it is more natural to consider all the extremal rays to study a Fano manifold since each extremal ray has various geometric information. We set up the following question.

Question 1.3. Give a bound of

$$
s(X):=\sum_{R \subset \mathrm{NE}(X) \text { extremal ray }}(l(R)-1)
$$

for arbitrary $n$-dimensional Fano manifolds $X$.

This question is a refinement of Conjectures 1.1 and 1.2 since the invariant $s(X)$ satisfies the inequality $\rho_{X}\left(r_{X}-1\right) \leq \rho_{X}\left(l_{X}-1\right) \leq s(X)$. We note that the invariant $s(X)$ is a natural invariant. For example, let $X:=\prod_{i=1}^{m} \mathbb{P}^{d_{i}}$ with $\sum_{i=1}^{m} d_{i}=n$. Then $s(X)=n$ holds despite $\rho_{X}\left(l_{X}-1\right)=m \cdot \min \left\{d_{i}\right\}$ is less than $n$ unless $d_{1}=\cdots=d_{m}$.

In this paper, we identify the bound of $s(X)$ when $n \leq 4$.

Theorem 1.4 (Main Theorem). Let $X$ be an n-dimensional Fano manifold.

(i) If $n \leq 3$, then $s(X) \leq n$ holds. Moreover, the equality holds if and only if

$$
X \simeq \prod_{R \subset \operatorname{NE}(X) \text { extremal ray }} \mathbb{P}^{l(R)-1} .
$$

(ii) If $n=4$, then $s(X) \leq n$ holds. Moreover, the equality holds if and only if

$$
X \simeq \prod_{R \subset \mathrm{NE}(X) \text { extremal ray }} \mathbb{P}^{l(R)-1}
$$

or

$$
X \simeq \mathrm{Bl}_{p, q}\left(\mathbb{Q}^{4}\right),
$$

the blowing up of $\mathbb{Q}^{4}$ along $p$ and $q$, where $\mathbb{Q}^{4} \subset \mathbb{P}^{5}$ is a smooth hyperquadric and $p, q$ are distinct points in $\mathbb{Q}^{4}$ with $\overline{p q} \not \subset \mathbb{Q}^{4}$, where $\overline{p q} \subset \mathbb{P}^{5}$ is the line through $p$ and $q$.

Remark 1.5. If $n \geq 5$, then there exsits an $n$-dimensional Fano manifold $X$ such that $s(X)$ is strictly larger than $n$ (see Remark 3.5 (iiii)). However, such $X$ is very special as far as we know. We think that all such $X$ should be classified.

As an immediate consequence of Theorem 1.4 we can give the affirmative answer to Conjecture 1.2 in the case $n \leq 4$. (Tsukioka Tsu12] proved the inequality in the case $n=4$ but did not settle the assertion on the equality case.)

Corollary 1.6 (cf. Tsu12]). Conjecture 1.2 is true if $n \leq 4$. 
Acknowledgements. The author would like to express his gratitude to Professor Shigefumi Mori for warm encouragements and valuable comments. He also thanks Professors Shigeru Mukai, Noboru Nakayama, Masayuki Kawakita and Stefan Helmke for valuable comments during the seminars in RIMS. The author thank the referee for useful comments. Especially, the proof of Theorem 1.4 (iii) has been improved by referee's comments. The author is partially supported by a JSPS Fellowship for Young Scientists. This paper is a modified version of the author's master thesis submitted in January 2011, to RIMS, Kyoto University.

Notation and terminology. We always work over the complex number field $\mathbb{C}$. For a proper variety $X$, let $\mathrm{N}_{1}(X)_{\mathbb{Q}}\left(\right.$ rep. $\left.\mathrm{N}^{1}(X)_{\mathbb{Q}}\right)$ be the vector space of onecycles (resp. Cartier divisors) on $X$, with rational coefficients, modulo numerical equivalence. Let $\mathrm{N}_{1}(X):=\mathrm{N}_{1}(X)_{\mathbb{Q}} \otimes_{\mathbb{Q}} \mathbb{R}$ and $\mathrm{N}^{1}(X):=\mathrm{N}^{1}(X)_{\mathbb{Q}} \otimes_{\mathbb{Q}} \mathbb{R}$. The Picard number of $X$, denoted by $\rho_{X}$, is defined to be the dimension of the vector space $\mathrm{N}_{1}(X)$.

For an $n$-dimensional normal projective variety $X$, we denote the normalization of the parameterizing space of irreducible and reduced rational curves on $X$ by RatCurves ${ }^{n}(X)$ (see Kol96, Definition II.2.11]). For the theory of extremal contraction, we refer the readers to KM98. A projective surjective morphism $f: X \rightarrow Z$ is called a contraction morphism if $Z$ is normal projective and any fiber of $f$ is connected. For an extremal ray $R \subset \overline{\mathrm{NE}}(X)$, we say that $R$ defines the contraction morphism $\operatorname{cont}_{R}: X \rightarrow Y$ if $\operatorname{cont}_{R}$ is a contraction morphism and the kernel of the surjection $\mathrm{N}_{1}(X) \rightarrow \mathrm{N}_{1}(Y)$ is equal to $\mathbb{R} R(=R+(-R))$. The morphism $\operatorname{cont}_{R}$ is called the associated contraction morphism. For example, if $X$ is smooth and $R$ is $K_{X}$-negative, then $R$ defines the contraction morphism. For an extremal ray $R \subset \overline{\mathrm{NE}}(X)$, we say that $R$ is of fiber type (resp. divisorial, small) if $R$ defines the contraction morphism $\operatorname{cont}_{R}: X \rightarrow Y$ and the morphism is of fiber type (resp. divisorial, small). We define

$$
\operatorname{Exc}(R):=\left\{x \in X \mid \operatorname{cont}_{R}: X \rightarrow Y \text { is not isomorphism at } x\right\} .
$$

For example, if $R$ is of fiber type, then $\operatorname{Exc}(R)=X$. We say that $R$ is of type $(a, b)$ if $\operatorname{dim}(\operatorname{Exc}(R))=a$ and $\operatorname{dim}\left(\operatorname{cont}_{R}(\operatorname{Exc}(R))\right)=b$, and we say that $R$ is of type $(n-1, b)^{\mathrm{sm}}$ if the associated contraction morphism is the blowing up morphism of a smooth projective variety along a smooth subvariety of dimension $b$ (in particular, $X$ must be smooth). For an extremal ray $R \subset \overline{\mathrm{NE}}(X)$ and a Cartier divisor $E$ on $X$, the notation $(E \cdot R)>0($ resp. $(E \cdot R)<0,(E \cdot R)=0)$ means that the property $(E \cdot C)>0$ (resp. $(E \cdot C)<0,(E \cdot C)=0)$ holds for a curve $C \subset X$ with $[C] \in R$.

For an algebraic variety $X$ and a closed subscheme $Y \subset X$, the morphism $\mathrm{Bl}_{Y}(X) \rightarrow X$ denotes the blowing up of $X$ along $Y$. The symbol $\mathbb{Q}^{n}$ denotes a smooth hyperquadric in $\mathbb{P}^{n+1}$. We say that $X$ is a Fano manifold if $X$ is a smooth projective variety such that the anticanonical divisor $-K_{X}$ is ample. 


\section{Preliminaries}

\subsection{A family of rational curves}

We observe the definition and a property of a family of rational curves for a fixed normal projective variety.

Definition 2.1 (see for example ACO04]). Let $X$ be a normal projective variety. We define a family of rational curves to be an irreducible component $H \subset$ RatCurves $^{n}(X)$. For any $x \in X$, let $H_{x}$ be the subvariety of $H$ parameterizing rational curves passing through $x$, and $\tilde{H}_{x}$ the normalization of the image of $H_{x}$ in the Chow variety $\operatorname{Chow}(X)$. We define $\operatorname{Locus}(H)$ (resp. $\operatorname{Locus}\left(H_{x}\right)$ ) to be the union of rational curves parameterized by $H$ (resp. $H_{x}$ ). For a family $H$ of rational curves on $X$, the family $H$ is said to be dominating if the closure $\overline{\operatorname{Locus}(H)}$ is equal to $X$, unsplit if $H$ is projective, and locally unsplit if $H_{x}$ is projective for general $x \in \operatorname{Locus}(H)$.

The following proposition may be familiar.

Proposition 2.2 ([NO10, Proposition 2.5(b)]). Let $X$ be a smooth projective variety, $H$ be a family of rational curves on $X$, and $x \in \operatorname{Locus}(H)$ be a point such that $H_{x}$ is projective. Then one has

$$
\operatorname{dim} \operatorname{Locus}(H)+\operatorname{dim} \operatorname{Locus}\left(H_{x}\right) \geq \operatorname{dim} X+\left(-K_{X} \cdot \operatorname{Fam} H\right)-1,
$$

where Fam $H$ is the numerical class of the curves in $X$ parametrized by $H$.

\subsection{Properties of extremal contractions}

We show some properties of extremal contractions associate to extremal rays that we need to prove Theorem 1.4.

Proposition 2.3. Let $X$ be an $n$-dimensional smooth projective variety. Assume that there exist distinct $K_{X}$-negative extremal rays $R_{1}, R_{2} \subset \overline{\mathrm{NE}}(X)$ such that $R_{1}$ is of type $(n-1,0), l\left(R_{2}\right) \geq 2$ and $\operatorname{Exc}\left(R_{1}\right) \cap \operatorname{Exc}\left(R_{2}\right) \neq \emptyset$. Then $R_{2}$ is of fiber type and $\rho_{X}=2$.

Proof. Let $E_{i}:=\operatorname{Exc}\left(R_{i}\right)$ for $i=1,2$ and fix $x \in E_{1} \cap E_{2}$. Let $C \subset X$ be a rational curve such that

(1) $x \in C$ and $[C] \in R_{2}$,

(2) $\left(-K_{X} \cdot C\right)$ is minimal among satisfying (1).

Let $H$ be a family of rational curves containing $[C] \in \operatorname{RatCurves}^{n}(X)$. Then $H_{x}$ is projective by construction. If there exists an irreducible curve $l \subset E_{1} \cap$ $\operatorname{Locus}\left(H_{x}\right)$ then $[l] \in R_{1} \cap R_{2}=\{0\}$, which leads to a contradiction. Hence $\operatorname{dim}\left(E_{1} \cap \operatorname{Locus}\left(H_{x}\right)\right)=0$. Thus $\operatorname{dim} \operatorname{Locus}\left(H_{x}\right) \leq 1$ since $\operatorname{dim} E_{1}=n-1$. Therefore,

$1 \geq \operatorname{dim} \operatorname{Locus}\left(H_{x}\right) \geq(n-\operatorname{dim} \operatorname{Locus}(H))+\left(-K_{X} \cdot \operatorname{Fam} H\right)-1$

$\geq l\left(R_{2}\right)-1 \geq 1$ 
by Proposition 2.2. Thus $\operatorname{dim} \operatorname{Locus}(H)=n$ and $l\left(R_{2}\right)=\left(-K_{X} \cdot \operatorname{Fam} H\right)=2$. In particular, $H$ is dominating and unsplit. Hence $R_{2}$ is of fiber type. Let $\varphi_{2}: X \rightarrow Y_{2}$ be the contraction morphism associated to $R_{2}$. Since the restriction $\left.\varphi_{2}\right|_{E_{1}}: E_{1} \rightarrow Y_{2}$ is a finite morphism, $\operatorname{dim} Y_{2}=n-1$. We note that all curves in $E_{1}$ are numerically proportional. Thus $\rho_{Y_{2}}=1$. This implies that $\rho_{X}=2$.

Proposition 2.4. Let $X$ be an $n$-dimensional normal projective variety which satisfies that $\operatorname{Pic}(X) \otimes \mathbb{Q}=\mathrm{N}^{1}(X)_{\mathbb{Q}}$.

(1) Assume that $\rho_{X} \geq 3$. Pick any extremal ray $R \subset \overline{\mathrm{NE}}(X)$ which defines the contraction morphism $\varphi: X \rightarrow Y$. Then the ray $R$ is neither of type $(n, 0)$ nor of type $(n, 1)$.

(2) Set $m \geq 2$. Let $R_{i} \subset \overline{\mathrm{NE}}(X)$ be an extremal ray which defines the contraction morphism $\varphi_{i}: X \rightarrow Y_{i}, C_{i} \subset X$ be an irreducible curve with $\left[C_{i}\right] \in R_{i}$, and $E_{i}:=\operatorname{Exc}\left(R_{i}\right)$ for any $1 \leq i \leq m$. We assume that $E_{i} \cap E_{j}=\emptyset$ for any $1 \leq i<j \leq m$. Then we can construct the morphism $\varphi: X \rightarrow Y$ contracting all of $E_{1}, \ldots, E_{m} . \quad\left(\right.$ Glue $\varphi_{1}, \ldots, \varphi_{m}$ together. We note that $Y$ is a normal proper variety but not necessary projective.) Then there is an exact sequence

$$
0 \longrightarrow \sum_{i=1}^{m} \mathbb{Q}\left[C_{i}\right] \longrightarrow \mathrm{N}_{1}(X)_{\mathbb{Q}} \stackrel{\varphi_{*}}{\longrightarrow} \mathrm{N}_{1}(Y)_{\mathbb{Q}} \longrightarrow 0 .
$$

Furthermore, if $X$ is $\mathbb{Q}$-factorial and $R_{i}$ is divisorial for any $1 \leq i \leq m$, then $Y$ is also $\mathbb{Q}$-factorial and hence $\rho_{Y} \geq 1$.

Proof. (11) is obvious. We prove (2). For $1 \leq i \leq m$, let $\psi_{i}: X \rightarrow Z_{i}$ be the morphism contracting $E_{1}, \ldots, E_{i}$ obtained by gluing $\varphi_{1}, \ldots, \varphi_{i}$ together (for construction, see [Har77, Exercise 2.12]). We note that $Z_{i}$ is a normal proper variety, $Y=Z_{m}$ and $\varphi=\psi_{m}$. Set $Z_{0}:=X$ and $\psi_{0}:=i d_{X}$ (the identity morphism). For $1 \leq i \leq m$, let $\pi_{i}: Z_{i-1} \rightarrow Z_{i}$ be the morphism contracting (the image of) $E_{i}$ such that $\pi_{i} \circ \psi_{i-1}=\psi_{i}$. We remark that $\varphi_{1}=\psi_{1}=\pi_{1}$. Note that $\operatorname{Pic}\left(Z_{i}\right) \otimes \mathbb{Q}=\mathrm{N}^{1}\left(Z_{i}\right)_{\mathbb{Q}}$ by Remark 2.5. It is enough to show the exactness of

$$
0 \longrightarrow \mathrm{N}^{1}\left(Z_{i}\right)_{\mathbb{Q}} \stackrel{\pi_{i}^{*}}{\longrightarrow} \mathrm{N}^{1}\left(Z_{i-1}\right)_{\mathbb{Q}} \stackrel{\left(\bullet \cdot C_{i}\right)}{\longrightarrow} \mathbb{Q}
$$

for any $1 \leq i \leq m$ to prove the exactness of the sequence in (2). We can assume that $2 \leq i \leq m$ since the case $i=1$ follows from the definition of the contraction morphism. The injectivity of $\pi_{i}^{*}: \mathrm{N}^{1}\left(Z_{i}\right)_{\mathbb{Q}} \rightarrow \mathrm{N}^{1}\left(Z_{i-1}\right)_{\mathbb{Q}}$ is obvious. Let $\tau_{i}: Y_{i} \rightarrow Z_{i}$ be the morphism contracting $E_{1}, \ldots, E_{i-1}$ which satisfies that the diagram commutes:

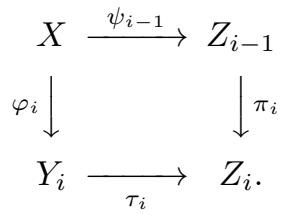

Let $V_{i}:=Z_{i} \backslash\left(\tau_{i} \circ \varphi_{i}\left(E_{1} \sqcup \ldots \sqcup E_{i-1}\right)\right)$ and $U_{i}:=Z_{i} \backslash\left(\tau_{i} \circ \varphi_{i}\left(E_{i}\right)\right)$. Pick any invertible sheaf $M \in \operatorname{Pic}\left(Z_{i-1}\right)$ satisfying $\left(M \cdot C_{i}\right)=0$. Then $0=\left(M \cdot C_{i}\right)=$ 
$\left(\psi_{i-1}{ }^{*} M \cdot C_{i}\right)$. There exists an invertible sheaf $L_{1} \in \operatorname{Pic}\left(Y_{i}\right)$ and a positive integer $t$ such that $\varphi_{i}{ }^{*} L_{1} \simeq \psi_{i-1}{ }^{*} M^{\otimes t}$ by the property of the ray $R_{i}$ and the fact $\operatorname{Pic}(X) \otimes \mathbb{Q}=\mathrm{N}^{1}(X)_{\mathbb{Q}}$. Thus

$$
M^{\otimes t} \simeq \psi_{i-1_{*}} \psi_{i-1}{ }^{*} M^{\otimes t} \simeq \psi_{i-1_{*}} \varphi_{i}^{*} L_{1} \simeq \pi_{i}^{*} \tau_{i *} L_{1}
$$

Indeed, $\varphi_{i}$ and $\pi_{i}$ are isomorphisms over $U_{i}$, and $\psi_{i-1}$ and $\tau_{i}$ are isomorphisms over $V_{i}$, respectively. We note that $\tau_{i *} L_{1}$ is an invertible sheaf since $\left.\tau_{i_{*}} L_{1}\right|_{U_{i}} \simeq$ $\left.M^{\otimes t}\right|_{\pi_{i}-1\left(U_{i}\right)}$ and $\left.\left.\tau_{i *} L_{1}\right|_{V_{i}} \simeq L_{1}\right|_{\tau_{i}-1\left(V_{i}\right)}$. Therefore we have $M^{\otimes t} \in \pi_{i}^{*}\left(\operatorname{Pic}\left(Z_{i}\right)\right)$. For the remaining part, see [KM98, Corollary 3.18] for example.

Remark 2.5. For a surjective morphism $\varphi: X \rightarrow Y$ between normal proper varieties with connected fibers, if $\operatorname{Pic}(X) \otimes \mathbb{Q}=\mathrm{N}^{1}(X)_{\mathbb{Q}}$ then $\operatorname{Pic}(Y) \otimes \mathbb{Q}=$ $\mathrm{N}^{1}(Y)_{\mathbb{Q}}$. Indeed, for a numerically trivial invertible sheaf $L \in \operatorname{Pic}(Y)$, since $\varphi^{*} L$ is numerically trivial, there exists a positive integer $t$ such that $\varphi^{*} L^{\otimes t} \simeq \mathcal{O}_{X}$. Thus $L^{\otimes t} \simeq \mathcal{O}_{Y}$.

Corollary 2.6. Let $X$ be an $n$-dimensional normal $\mathbb{Q}$-factorial projective variety such that $\operatorname{Pic}(X) \otimes \mathbb{Q}=\mathrm{N}^{1}(X)_{\mathbb{Q}}$. Assume that there exist distinct divisorial extremal rays $R_{1}, \ldots, R_{m} \subset \overline{\mathrm{NE}}(X)$ which define the contraction morphisms $\varphi_{i}: X \rightarrow Y_{i}$ for all $1 \leq i \leq m$ and $\operatorname{Exc}\left(R_{i}\right) \cap \operatorname{Exc}\left(R_{j}\right)=\emptyset$ for any $1 \leq i<j \leq m$.

(1) If $m \geq 3$, then $\rho_{X} \geq 4$.

(2) If $X$ is smooth and $R_{i}$ is of type $\left(n-1, b_{i}\right)^{\mathrm{sm}}$ (for some $b_{i} \in \mathbb{Z}_{\geq 0}$ ) for any $1 \leq i \leq m$, then $\rho_{X} \geq m+1$.

Proof. Let $\varphi: X \rightarrow Y$ be the morphism which is the gluing morphism of $\varphi_{1}, \ldots, \varphi_{m}$ contracting $\operatorname{Exc}\left(R_{1}\right), \ldots, \operatorname{Exc}\left(R_{m}\right)$ as in Proposition 2.4 (2). Let $C_{i} \subset X$ be an irreducible and reduced curve with $\left[C_{i}\right] \in R_{i}$ for $1 \leq i \leq m$.

(11) We can assume that the classes $\left[C_{1}\right],\left[C_{2}\right],\left[C_{3}\right]$ are linearly independent in $\mathrm{N}_{1}(X)$. By Proposition 2.4 (2), $Y$ is $\mathbb{Q}$-factorial and $1 \leq \rho_{Y} \leq \rho_{X}-3$.

(2) In this case, $Y$ is a smooth proper variety and $\rho_{X}=m+\rho_{Y} \geq m+1$.

We recall Wiśniewski's theorem on the bounds of the length of extremal rays.

Theorem 2.7 (Wiś91, Theorem 1.1]). Let $X$ be a smooth projective variety, $R \in \overline{\mathrm{NE}}(X)$ be a $K_{X}$-negative extremal ray and $\operatorname{cont}_{R}: X \rightarrow Y$ be the associated contraction morphism. Then for every irreducible component $E \subset \operatorname{Exc}(R)$, we have

$$
l(R) \leq \operatorname{dim} X+1-2 \operatorname{codim}_{X} E-\operatorname{dim}\left(\operatorname{cont}_{R}(E)\right) .
$$

\subsection{Characterizations of the products of projective spaces}

We give several criteria so that a given smooth projective variety is isomorphic to the products of projective spaces. 
Theorem 2.8 ([Keb02, Theorem 2.16]). Let $X$ be a normal projective variety and $H$ be a dominating and locally unsplit family of rational curves on $X$. For general $x \in X$, consider the rational map

$$
\tau_{x}: \tilde{H}_{x} \rightarrow \mathbb{P}\left(\left.T_{X}\right|_{x} ^{\vee}\right)
$$

defined by

$$
[l] \mapsto \mathbb{P}\left(\left.T_{l}\right|_{x} ^{\vee}\right) .
$$

Then the rational map $\tau_{x}$ is a finite morphism.

Definition 2.9 (Variety of Minimal Rational Tangents). Under the assumption in Theorem 2.8, the finite morphism $\tau_{x}$ is called the tangent morphism; its image $\mathcal{C}_{x}:=\tau_{x}\left(\tilde{H}_{x}\right) \subset \mathbb{P}\left(\left.T_{X}\right|_{x} ^{\vee}\right)$ is called the variety of minimal rational tangents, or shortly VMRT, of $H$ at $x$.

Araujo Ara06] showed a criterion for varieties being isomorphic to the products of projective spaces in terms of VMRT.

Theorem 2.10 (Ara06, Theorem 1.3]). Let $X$ be an $n$-dimensional smooth projective variety with $k$ distinct dominating and unsplit family of rational curves $H_{1}, \ldots, H_{k}$ on $X$. Suppose that, for a general $x \in X$, the associated VMRT of $H_{i}$ at $x$ are linear subspaces of dimension $d_{i}-1$ in $\mathbb{P}\left(\left.T_{X}\right|_{x} ^{\vee}\right)$ such that $\sum_{i=1}^{k} d_{i}=$ n. Then $X \simeq \prod_{i=1}^{k} \mathbb{P}^{d_{i}}$.

We give another criterion for varieties being isomorphic to the products of projective spaces in terms of length of extremal rays.

Theorem 2.11. Let $X$ be an $n$-dimensional smooth projective variety with $n=$ $\sum_{i=1}^{k} d_{i}$, where $d_{1}, \ldots, d_{k} \in \mathbb{Z}_{>0}$. Assume that there exist distinct $K_{X}$-negative extremal rays $R_{1}, \ldots, R_{k} \subset \overline{\mathrm{NE}}(X)$ such that $R_{i}$ are of fiber type and $l\left(R_{i}\right) \geq$ $d_{i}+1$ for all $1 \leq i \leq k$. Then $X \simeq \prod_{i=1}^{k} \mathbb{P}^{d_{i}}$.

Proof. Let $\varphi_{i}: X \rightarrow Y_{i}$ be the contraction morphism associated to $R_{i}$ and $e_{i}:=$ $\operatorname{dim} X-\operatorname{dim} Y_{i}$ for $1 \leq i \leq k$. We have $\sum_{i=1}^{k} e_{i} \leq n$ and $e_{i} \geq l\left(R_{i}\right)-1$ for any $i$ by Wiś91, Theorem 2.2] and Theorem [2.7. Hence we obtain the inequality

$$
n \geq \sum_{i=1}^{k} e_{i} \geq \sum_{i=1}^{k}\left(l\left(R_{i}\right)-1\right) \geq \sum_{i=1}^{k} d_{i}=n .
$$

Therefore $e_{i}=l\left(R_{i}\right)-1=d_{i}$ for any $i$. Let $F_{i}$ be a general fiber of $\varphi_{i}$. Then $F_{i}$ is a $d_{i}$-dimensional Fano manifold such that any rational curve $l_{i}$ in $F_{i}$ satisfies that $\left(-K_{F_{i}} \cdot l_{i}\right) \geq d_{i}+1$. Hence $F_{i} \simeq \mathbb{P}^{d_{i}}$ by CMSB02. Let $H_{i}$ be the family of rational curves on $X$ containing points parameterizing lines in $F_{i} \simeq \mathbb{P}^{d_{i}}$. Then $H_{i}$ is a dominating and unsplit family since $\left(-K_{X} \cdot\right.$ Fam $\left.H_{i}\right)=d_{i}+1=l\left(R_{i}\right)$. We consider $\mathcal{C}_{x}^{i} \subset \mathbb{P}\left(\left.T_{X}\right|_{x} ^{\vee}\right)$ for $x \in F_{i}$, which is a VMRT of $H_{i}$ at $x$. We have $\mathcal{C}_{x}^{i}=\mathbb{P}\left(\left.T_{F_{i}}\right|_{x} ^{\vee}\right) \subset \mathbb{P}\left(\left.T_{X}\right|_{x} ^{\vee}\right)$; a linear subspace of dimension $d_{i}-1$. By Theorem 2.10. $X \simeq \prod_{i=1}^{k} \mathbb{P}^{d_{i}}$. 
We also give a criterion for varieties being isomorphic to the product of two projective spaces in terms of extremal rays.

Proposition 2.12. Let $X$ be an $n$-dimensional smooth projective variety. If there exist distinct $K_{X}$-negative extremal rays $R_{1}, R_{2} \subset \overline{\mathrm{NE}}(X)$ such that the intersection $\operatorname{Exc}\left(R_{1}\right) \cap \operatorname{Exc}\left(R_{2}\right)$ is not empty. Then we have

$$
\left(l\left(R_{1}\right)-1\right)+\left(l\left(R_{2}\right)-1\right) \leq n,
$$

and the equality holds if and only if $X \simeq \mathbb{P}^{l\left(R_{1}\right)-1} \times \mathbb{P}^{l\left(R_{2}\right)-1}$.

Proof. We fix an arbitrary point $x \in \operatorname{Exc}\left(R_{1}\right) \cap \operatorname{Exc}\left(R_{2}\right)$. For $i=1,2$, let $\varphi_{i}: X \rightarrow Y_{i}$ be the contraction morphism associated to $R_{i}$ and set $y_{i}:=\varphi_{i}(x) \in$ $Y_{i}$. Let $C_{i} \subset X$ be a rational curve which satisfies that

(1) $x \in C_{i}$ and $\left[C_{i}\right] \in R_{i}$,

(2) $\left(-K_{X} \cdot C_{i}\right)$ is minimal among satisfying (1).

Let $H_{i}$ be a family of rational curves on $X$ containing $\left[C_{i}\right] \in \operatorname{RatCurves}^{n}(X)$. Then $\left(H_{i}\right)_{x}$ is projective by construction. Hence we have

$$
\begin{aligned}
\operatorname{dim} \varphi_{i}^{-1}\left(y_{i}\right) & \geq \operatorname{dim} \operatorname{Locus}\left(\left(H_{i}\right)_{x}\right) \\
& \geq\left(n-\operatorname{dim} \operatorname{Locus}\left(H_{i}\right)\right)+\left(-K_{X} \cdot \operatorname{Fam} H_{i}\right)-1 \\
& \geq\left(-K_{X} \cdot \operatorname{Fam} H_{i}\right)-1 \geq l\left(R_{i}\right)-1
\end{aligned}
$$

by Proposition 2.2. We note that the intersection $\varphi_{1}^{-1}\left(y_{1}\right) \cap \varphi_{2}^{-1}\left(y_{2}\right)$ does not contain curves since the rays $R_{1}$ and $R_{2}$ are distinct. Hence $\operatorname{dim}\left(\varphi_{1}^{-1}\left(y_{1}\right) \cap\right.$ $\left.\varphi_{2}^{-1}\left(y_{2}\right)\right)=0$. Thus $n \geq \operatorname{dim} \varphi_{1}^{-1}\left(y_{1}\right)+\operatorname{dim} \varphi_{2}^{-1}\left(y_{2}\right)$. Hence $n \geq\left(l\left(R_{1}\right)-1\right)+$ $\left(l\left(R_{2}\right)-1\right)$. If $n=\left(l\left(R_{1}\right)-1\right)+\left(l\left(R_{2}\right)-1\right)$, then $H_{i}$ is dominating and unsplit for each $i=1,2$ since $\left(-K_{X} \cdot \operatorname{Fam} H_{i}\right)=l\left(R_{i}\right)$ and $\operatorname{dim} \operatorname{Locus}\left(H_{i}\right)=n$. Therefore one has $X \simeq \mathbb{P}^{l\left(R_{1}\right)-1} \times \mathbb{P}^{l\left(R_{2}\right)-1}$ by [cc06, Theorem 1.1].

Corollary 2.13. Let $X$ be an $n$-dimensional Fano manifold with $\rho_{X}=2$. Then $\mathrm{NE}(X)$ is spanned by two extremal rays, say $R_{1}$ and $R_{2}$. If, at least, one of $R_{1}$ and $R_{2}$ is not small, then we have

$$
\left(l\left(R_{1}\right)-1\right)+\left(l\left(R_{2}\right)-1\right) \leq n,
$$

and the equality holds if and only if $X \simeq \mathbb{P}^{l\left(R_{1}\right)-1} \times \mathbb{P}^{l\left(R_{2}\right)-1}$.

Proof. For $i=1,2$, let $\varphi_{i}: X \rightarrow Y_{i}$ be the contraction morphism associated to $R_{i}$ and $E_{i}:=\operatorname{Exc}\left(R_{i}\right)$. It is enough to show that $E_{1} \cap E_{2} \neq \emptyset$ by Proposition 2.12. We can assume that $R_{1}$ is divisorial. Then we have $\left(E_{1} \cdot R_{1}\right)<0$. Thus $\left(E_{1} \cdot R_{2}\right)>0$ holds since $E_{1}$ is a prime divisor and since $R_{1}$ and $R_{2}$ span the cone $\operatorname{NE}(X)$. Hence $E_{1} \cap E_{2} \neq \emptyset$. 


\section{$3 \quad$ Fano manifolds having special extremal rays}

In this section, we see several classification results of Fano manifolds having special extremal rays and calculate $s(X)$ for such Fano manifolds $X$.

Theorem 3.1 (Cas09, Proposition 3.1 and Theorem 1.1]). Let $X$ be an $n$ dimensional Fano manifold and $R \subset \mathrm{NE}(X)$ be an extremal ray.

(1) If $n \geq 3$ and $R$ is of type $(n-1,0)$, then $\rho_{X} \leq 3$.

(2) If $n \geq 4$ and $R$ is of type $(n-1,1)$, then $\rho_{X} \leq 5$.

Theorem 3.2 ([AO02, Theorem 5.1]). Let $X$ be an n-dimensional smooth projective variety and $R \subset \overline{\mathrm{NE}}(X)$ be a $K_{X}$-negative extremal ray of type $(n-1, m)$ which satisfies that $l(R)=n-1-m$ and all nontrivial fibers of the associated contraction morphism of $R$ are of equi-dimensional. Then $R$ is of type $(n-1, m)^{\mathrm{sm}}$.

Proposition 3.3 ([Tsu10b, Proposition 5] (and [AO02, Theorem 5.1])). Let $X$ be an $n$-dimensional Fano manifold with $n \geq 4$. Assume that there exist distinct extremal rays $R_{1}, R_{2} \subset \mathrm{NE}(X)$ such that $R_{i}$ is of type $(n-1,1)$ and $l\left(R_{i}\right)=n-2$ for each $i=1,2$. Then $\operatorname{Exc}\left(R_{1}\right) \cap \operatorname{Exc}\left(R_{2}\right)=\emptyset$.

Theorem 3.4 ([BCW02, Theorem 1.1]). Let $Y$ be an $n$-dimensional smooth projective variety with $n \geq 3$ and $a \in Y$ be a (closed) point. Then $X:=\mathrm{Bl}_{a}(Y)$ is a Fano manifold if and only if one of the following holds:

(i) $Y \simeq \mathbb{P}^{n}$ and $a \in Y$ is an arbitrary point.

(ii) $Y \simeq \mathbb{Q}^{n}$ and $a \in Y$ is an arbitrary point.

(iii) $Y \simeq V_{d}$ with $1 \leq d \leq n$ and $a \notin H^{\prime}$ (the strict transform of $H$ ) with $V_{d}:=\mathrm{Bl}_{Z}\left(\mathbb{P}^{n}\right)$, where $H \subset \mathbb{P}^{n}$ is a hyperplane and $Z \subset H$ is a smooth subvariety of dimension $n-2$ and degree $d$.

Remark 3.5. We have the following properties by easy calculations.

(i) If $X=\mathrm{Bl}_{a}(Y)$ is in Theorem 3.4 (ii), then

$$
\begin{aligned}
\mathrm{NE}(X) & =\mathbb{R}_{\geq 0}[f]+\mathbb{R}_{\geq 0}[g], \\
\left(-K_{X} \cdot f\right) & =2, \\
\left(-K_{X} \cdot g\right) & =n-1
\end{aligned}
$$

hold, where $f$ is the strict transform of a line on $Y=\mathbb{P}^{n}$ passing through $a$ and $g$ is a line in the exceptional divisor $\left(\simeq \mathbb{P}^{n-1}\right)$ of $X \rightarrow Y$. Thus $s(X)=n-1$.

(ii) If $X=\mathrm{Bl}_{a}(Y)$ is in Theorem 3.4 (iii), then

$$
\begin{aligned}
\mathrm{NE}(X) & =\mathbb{R}_{\geq 0}[f]+\mathbb{R}_{\geq 0}[g], \\
\left(-K_{X} \cdot f\right) & =1 \\
\left(-K_{X} \cdot g\right) & =n-1
\end{aligned}
$$


hold, where $f$ is the strict transform of a line on $Y=\mathbb{Q}^{n}$ passing through $a$ and $g$ is a line in the exceptional divisor $\left(\simeq \mathbb{P}^{n-1}\right)$ of $X \rightarrow Y$. Thus $s(X)=n-2$.

(iii) If $X=\mathrm{Bl}_{a}(Y)$ is in Theorem 3.4 (iii), then

$$
\begin{aligned}
\mathrm{NE}(X) & =\mathbb{R}_{\geq 0}[f]+\mathbb{R}_{\geq 0}[g]+\mathbb{R}_{\geq 0}[l]+\mathbb{R}_{\geq 0}[m], \\
l & \equiv m+g+(1-d) f \text { in } \mathrm{N}_{1}(X), \\
\left(-K_{X} \cdot f\right) & =1, \quad\left(-K_{X} \cdot g\right)=1, \\
\left(-K_{X} \cdot l\right) & =n+1-d, \quad\left(-K_{X} \cdot m\right)=1
\end{aligned}
$$

hold, where $f \subset X$ is a fiber over $Z, g \subset X$ is a line in a fiber over $a$, $l \subset X$ is a line in $H^{\prime}$, and $m \subset X$ is a strict transform of a line passing through $a$ and a point in $Z$. Thus if $d=1$ then $s(X)=n-2$, but if $d>1$ then $s(X)=2 n-2-d$. We note that if $d=2$, then $X$ is isomorphic to $\mathrm{Bl}_{p, q}\left(\mathbb{Q}^{n}\right)$ with $\overline{p q} \not \subset \mathbb{Q}^{n}\left(\subset \mathbb{P}^{n+1}\right)$ (see [BCW02, Corollaire 1.2]) and $s(X)=2 n-4$.

Theorem 3.6 (Cas08, Tsu10a, Tsu12]). Let $Y$ be an $n$-dimensional smooth projective variety with $n \geq 4, C \subset Y$ be a smooth curve, $X:=\mathrm{Bl}_{C}(Y)$, and $E$ be the exceptional divisor of the morphism $X \rightarrow Y$. We assume that $X$ is a Fano manifold.

(1) If $\rho_{X}=5$, then one of the following holds:

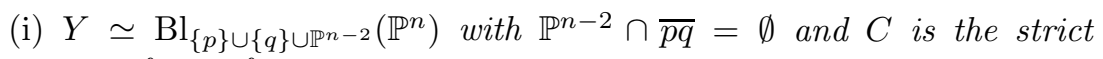
transform of $\overline{p q}$.

(ii) $Y \simeq \operatorname{Bl}_{\{p\} \cup\{q\} \cup \mathbb{Q}^{n-2}}\left(\mathbb{P}^{n}\right)$ with $\mathbb{Q}^{n-2} \cap \overline{p q}=\emptyset$ and $C$ is the strict transform of $\overline{p q}$.

(2) Assume that there exists an extremal ray $R \subset \mathrm{NE}(X)$ of fiber type with $l(R) \geq 2$ and $(E \cdot R)>0$.

- If $R$ is of type $(n, n-2)$, then $\rho_{X}=2$.

- If $R$ is of type $(n, n-1)$, then the pair of $(Y, C)$ is one of the following:

(i) $Y \simeq \mathbb{Q}^{n}$ and $C$ is a line in $\mathbb{Q}^{n} \subset \mathbb{P}^{n+1}$.

(ii) $Y \simeq \mathbb{P}^{1} \times \mathbb{P}^{n-1}$ and $C$ is a fiber of the second projection.

(iii) $Y \simeq \mathrm{Bl}_{\mathbb{P}^{n-2}}\left(\mathbb{P}^{n}\right)$ and $C$ is the strict transform of a line in $\mathbb{P}^{n}$ disjoint from $\mathbb{P}^{n-2}$.

(iv) $Y \simeq \mathrm{Bl}_{\mathbb{P}^{n-2}}\left(\mathbb{P}^{n}\right)$ and $C$ is a fiber of the blowing up.

(v) $Y \simeq \mathbb{P}_{\mathbb{P}^{1}}\left(\mathcal{O}_{\mathbb{P}^{1}} \oplus \mathcal{O}_{\mathbb{P}^{1}}(1)^{\oplus n-1}\right)$ and $C$ is the section of $\mathbb{P}^{n-1}$ bundle over $\mathbb{P}^{1}$ whose normal bundle $\mathcal{N}_{C / Y}$ is isomorphic to $\mathcal{O}_{\mathbb{P}^{1}}(-1)^{\oplus n-1}$.

(3) Assume that there exists an extremal ray $R \subset \mathrm{NE}(X)$ of fiber type with $(E \cdot R)=0$. Let $\varphi: X \rightarrow Z$ be the contraction morphism associated to $R$. 
Then $R$ is of type $(n, n-1), C \simeq \mathbb{P}^{1}, E \simeq \mathbb{P}^{1} \times \mathbb{P}^{n-2}, E=\varphi^{*} D$ and $Z$ is factorial, where $D:=\varphi(E)$ with the reduced structure. Furthermore, if $n=4$, then there exists an extremal ray $R_{Z} \subset \mathrm{NE}(Z)$ with the associated contraction morphism $\varphi_{Z}: Z \rightarrow W$ such that $\varphi_{Z}$ maps $D$ to a point.

Proof. (11) and (2) follow from [Tsu10a, Theorem 1] and [Tsu12, Propositions $3,4]$. We prove (3). The ray $R$ is of type $(n, n-1), E=\varphi^{*} D$ and $Z$ is factorial by the fact $\operatorname{dim} D \geq n-2$ and by Cas08, Lemmas 3.9 (i), 3.10 (i)]. Moreover, $C \simeq \mathbb{P}^{1}$ since a one-dimensional fiber of $\varphi$ in $E$ maps $X \rightarrow Y$ onto $C$. We know that $E \simeq \mathbb{P}^{1} \times \mathbb{P}^{n-2}$ since $E \simeq \mathbb{P}_{C}\left(\mathcal{N}_{C / Y}^{\vee}\right)$ and $\operatorname{dim} E>\operatorname{dim} D$, where $\mathcal{N}_{C / Y}$ is the normal bundle of $C$ in $Y$. The cone $\mathrm{NE}(Z)$ is closed since $\mathrm{NE}(X)$ is so. Assume that $n=4$. Then the existence of the ray $R_{Z} \subset \mathrm{NE}(Z)$ follows from Cas08, Theorem 4.1 (ii)].

Remark 3.7. We have the following properties by easy calculations.

(i) If $X=\mathrm{Bl}_{C}(Y)$ is in Theorem 3.6 (11) (i), then

$$
\begin{aligned}
\operatorname{NE}(X) & =\mathbb{R}_{\geq 0}[e]+\mathbb{R}_{\geq 0}[f]+\mathbb{R}_{\geq 0}[g]+\mathbb{R}_{\geq 0}[h] \\
& +\mathbb{R}_{\geq 0}[k]+\mathbb{R}_{\geq 0}[l]+\mathbb{R}_{\geq 0}[m], \\
\left(-K_{X} \cdot e\right) & =n-2, \quad\left(-K_{X} \cdot f\right)=1, \quad\left(-K_{X} \cdot g\right)=1, \\
\left(-K_{X} \cdot h\right) & =1,\left(-K_{X} \cdot k\right)=1,\left(-K_{X} \cdot l\right)=1,\left(-K_{X} \cdot m\right)=1,
\end{aligned}
$$

and $\operatorname{NE}(X)$ is exactly spanned by the above seven rays, where

- $e$ is a nontrivial fiber of the morphism $X \rightarrow Y$,

- $f$ is the strict transform of a line in the exceptional divisor over $p$,

- $g$ is the strict transform of a line in the exceptional divisor over $q$,

- $h$ is a fiber over $\mathbb{P}^{n-2}$,

- $k$ is a fiber of $E \simeq C \times \mathbb{P}^{n-2} \rightarrow \mathbb{P}^{n-2}$, where $E$ is the exceptional divisor of $X \rightarrow Y$,

- $l$ is the strict transform of a line in $\mathbb{P}^{n}$ passing through $p$ and $\mathbb{P}^{n-2}$,

- $m$ is the strict transform of a line in $\mathbb{P}^{n}$ passing through $q$ and $\mathbb{P}^{n-2}$.

Thus $s(X)=n-3$.

(ii) If $X=\mathrm{Bl}_{C}(Y)$ is in Theorem 3.6 (11) (ii), then

$$
\begin{aligned}
\mathrm{NE}(X) & =\mathbb{R}_{\geq 0}[e]+\mathbb{R}_{\geq 0}[f]+\mathbb{R}_{\geq 0}[g]+\mathbb{R}_{\geq 0}[h] \\
& +\mathbb{R}_{\geq 0}[j]+\mathbb{R}_{\geq 0}[k]+\mathbb{R}_{\geq 0}[l]+\mathbb{R}_{\geq 0}[m], \\
\left(-K_{X} \cdot e\right) & =n-2,\left(-K_{X} \cdot f\right)=1,\left(-K_{X} \cdot g\right)=1,\left(-K_{X} \cdot h\right)=1, \\
\left(-K_{X} \cdot j\right) & =1,\left(-K_{X} \cdot k\right)=1,\left(-K_{X} \cdot l\right)=1,\left(-K_{X} \cdot m\right)=1,
\end{aligned}
$$

and $\mathrm{NE}(X)$ is exactly spanned by the above eight rays, where 
- $e$ is a nontrivial fiber of the morphism $X \rightarrow Y$,

- $f$ is the strict transform of a line in the exceptional divisor over $p$,

- $g$ is the strict transform of a line in the exceptional divisor over $q$,

- $h$ is a fiber over $\mathbb{Q}^{n-2}$,

- $j$ is the strict transform of a line in $\mathbb{P}^{n}$ intersects $\overline{p q}$ with each other and is contained in a unique hyperplane in $\mathbb{P}^{n}$ which contains $\mathbb{Q}^{n-2}$,

- $k$ is a fiber of $E \simeq C \times \mathbb{P}^{n-2} \rightarrow \mathbb{P}^{n-2}$, where $E$ is the exceptional divisor of $X \rightarrow Y$,

- $l$ is the strict transform of a line in $\mathbb{P}^{n}$ passing through $p$ and $\mathbb{Q}^{n-2}$,

- $m$ is the strict transform of a line in $\mathbb{P}^{n}$ passing through $q$ and $\mathbb{Q}^{n-2}$.

Thus $s(X)=n-3$.

(2) (i) If $X=\mathrm{Bl}_{C}(Y)$ is in Theorem3.6(2) (i), then $\rho_{X}=2$. Thus $s(X)<n$ by Corollary 2.13 .

(ii) If $X=\mathrm{Bl}_{C}(Y)$ is in Theorem 3.6 (2) (ii), then

$$
\begin{aligned}
\mathrm{NE}(X) & =\mathbb{R}_{\geq 0}[f]+\mathbb{R}_{\geq 0}[g]+\mathbb{R}_{\geq 0}[h], \\
\left(-K_{X} \cdot f\right) & =n-2, \quad\left(-K_{X} \cdot g\right)=2, \quad\left(-K_{X} \cdot h\right)=2
\end{aligned}
$$

hold, where $f$ is a nontrivial fiber of $X \rightarrow Y, g$ is the strict transform of a general fiber of the first projection $Y=\mathbb{P}^{1} \times \mathbb{P}^{n-1} \rightarrow \mathbb{P}^{n-1}$ and $h$ is the strict transform of a line in the second projection $Y=$ $\mathbb{P}^{1} \times \mathbb{P}^{n-1} \rightarrow \mathbb{P}^{1}$ passing through $C$. Thus $s(X)=n-1$.

(iii) If $X=\mathrm{Bl}_{C}(Y)$ is in Theorem 3.6 (2) (iii), then

$$
\begin{aligned}
\mathrm{NE}(X) & =\mathbb{R}_{\geq 0}[f]+\mathbb{R}_{\geq 0}[g]+\mathbb{R}_{\geq 0}[h], \\
\left(-K_{X} \cdot f\right) & =n-2, \quad\left(-K_{X} \cdot g\right)=1, \quad\left(-K_{X} \cdot h\right)=2
\end{aligned}
$$

hold, where $f$ is a nontrivial fiber of $X \rightarrow Y, g$ is a fiber over $\mathbb{P}^{n-2}$ and $h$ is the strict transform of a line in $\mathbb{P}^{n}$ passing through $C$ and $\mathbb{P}^{n-2}$. Thus $s(X)=n-2$.

(iv) If $X=\mathrm{Bl}_{C}(Y)$ is in Theorem 3.6 (2) (iv), then

$$
\begin{aligned}
\mathrm{NE}(X) & =\mathbb{R}_{\geq 0}[f]+\mathbb{R}_{\geq 0}[g]+\mathbb{R}_{\geq 0}[h], \\
\left(-K_{X} \cdot f\right) & =n-2, \quad\left(-K_{X} \cdot g\right)=1, \quad\left(-K_{X} \cdot h\right)=2
\end{aligned}
$$

hold, where $f$ is a nontrivial fiber of $X \rightarrow Y, g$ is a general fiber over $\mathbb{P}^{n-2}$ and $h$ is the strict transform of a line in $\mathbb{P}^{n}$ passing through $\mathbb{P}^{n-2}$ and the image of $C$ in $\mathbb{P}^{n}$. Thus $s(X)=n-2$. 
(v) If $X=\mathrm{Bl}_{C}(Y)$ is in Theorem 3.6 (2) (v), then

$$
\begin{aligned}
\mathrm{NE}(X) & =\mathbb{R}_{\geq 0}[f]+\mathbb{R}_{\geq 0}[g]+\mathbb{R}_{\geq 0}[h], \\
\left(-K_{X} \cdot f\right) & =n-2, \quad\left(-K_{X} \cdot g\right)=1, \quad\left(-K_{X} \cdot h\right)=2
\end{aligned}
$$

hold, where $f$ is a nontrivial fiber of $X \rightarrow Y, g$ is a fiber of $E \simeq$ $C \times \mathbb{P}^{n-2} \rightarrow \mathbb{P}^{n-2}$, where $E$ is the exceptional divisor of $X \rightarrow Y$, and $h$ is the strict transform of a line in a fiber of $Y \rightarrow \mathbb{P}^{1}$ passing through $C$. Thus $s(X)=n-2$.

\section{Proof of Theorem 1.4}

In this section, we prove Theorem 1.4. If an $n$-dimensional Fano manifold $X$ satisfies that $s(X) \geq n$ and $\rho_{X}=1$, then $s(X)=n$ and $X \simeq \mathbb{P}^{n}$ by [CMSB02. Hence we can consider only the Fano manifolds $X$ with $\rho_{X} \geq 2$.

\subsection{Proof of Theorem 1.4 (i)}

We can assume that $n=3$ since the case $n \leq 2$ is trivial. We prove the assertion without using the result [MM81] of complete classification of 3-dimensional Fano manifolds $X$ with $\rho_{X} \geq 2$. Let $X$ be a 3 -dimensional Fano manifold with $s(X) \geq 3$. We can assume that $\rho_{X} \geq 3$ by Corollary 2.13. By Theorem 2.7. Proposition 2.4 (1) and Theorem 3.2 any extremal ray $R \subset \mathrm{NE}(X)$ with $l(R) \geq 2$ satisfies one of the following:

(A) $R$ is of type $(2,0)^{\mathrm{sm}}$ and $l(R)=2$.

(B) $R$ is of type $(3,2)$ and $l(R)=2$.

(We note that this result directly follows from Mor82, Theorems 3.3, 3.5].) If there exists an extremal ray $R \subset \mathrm{NE}(X)$ of type (A), then $X \simeq \mathrm{Bl}_{a}\left(V_{d}\right)$ with $1 \leq d \leq 3$ by Theorem 3.4, thus $s(X)<3$ by Remark 3.5 (iii). If there exist distinct extremal rays $R_{1}, R_{2}$ and $R_{3} \subset \mathrm{NE}(X)$ such that all of them are of type (B), then $X \simeq \mathbb{P}^{1} \times \mathbb{P}^{1} \times \mathbb{P}^{1}$ by Theorem 2.11, Therefore we have completed the proof of Theorem 1.4 (i).

\subsection{Proof of Theorem 1.4 (iii)}

Let $X$ be a 4-dimensional Fano manifold with $s(X) \geq 4$. We can assume that $\rho_{X} \geq 3$ by Corollary 2.13. (We note that if $\rho_{X}=2$ and both extremal rays are small, then $s(X)=0$.) By Theorem 2.7. Proposition 2.4 (11) and Theorem 3.2 . any extremal ray $R \subset \mathrm{NE}(X)$ with $l(R) \geq 2$ satisfies one of the following:

(A) $R$ is of type $(3,0)^{\mathrm{sm}}$ and $l(R)=3$.

(B) $R$ is of type $(3,0)$ and $l(R)=2$.

(C) $R$ is of type $(3,1)^{\mathrm{sm}}$ and $l(R)=2$. 
(D) $R$ is of type $(4,3)$ and $l(R)=2$.

(E) $R$ is of type $(4,2)$ and $l(R)=3$.

(F) $R$ is of type $(4,2)$ and $l(R)=2$.

We note that all two distinct divisorial extremal rays $R_{1}, R_{2}$ with $l\left(R_{1}\right), l\left(R_{2}\right) \geq$ 2 satisfy that $\operatorname{Exc}\left(R_{1}\right) \cap \operatorname{Exc}\left(R_{2}\right)=\emptyset$ by Propositions 2.3 and 3.3 .

Assume that there exists an extremal ray $R$ of type (A). Then $X \simeq$ $\mathrm{Bl}_{a}\left(V_{2}\right) \simeq \mathrm{Bl}_{p, q}\left(\mathbb{Q}^{4}\right)$ and $s(X)=4$ by Theorem 3.4 and Remark 3.5 (iii). Assume that there exists an extremal ray $R$ of type $(\mathrm{B})$ and there is no extremal ray of type (A). Then $\rho_{X}=3$ and any other extremal ray $R^{\prime}$ with $l\left(R^{\prime}\right) \geq 2$ is of type (B) or (C) by Proposition 2.3 and Theorem 3.1 (11). Since $s(X) \geq 4$, there exist distinct extremal rays $R_{1}, R_{2}, R_{3}$ apart from $R$ such that each of them is of type (B) or (C). This contradicts to Corollary 2.6 (11). Hence we can assume that any extremal ray $R$ with $l(R) \geq 2$ is of type (C), (D), (E) or (F).

Assume that there exists an extremal ray $R_{1}$ of type (C). We have $\rho_{X} \leq 4$ by Theorems 3.1 (2), 3.6 (11) and Remark 3.7 (11). By Corollary 2.6 (11), the number of extremal rays of type $(\mathrm{C})$ is at most three. Since $s(X) \geq 4$, there exists an extremal ray $R_{0}$ of fiber type and $l\left(R_{0}\right) \geq 2$. Then $\left(\operatorname{Exc}\left(R_{1}\right) \cdot R_{0}\right)=0$ and $R_{0}$ is of type (D) by Theorem 3.6 (2), (3) and Remark 3.7 (2). Moreover, any extremal ray $R^{\prime}$ of fiber type apart from $R_{0}$ satisfies that $\left(\operatorname{Exc}\left(R_{1}\right) \cdot R^{\prime}\right)>0$. Indeed, by Theorem 3.6 (3), if $\left(\operatorname{Exc}\left(R_{1}\right) \cdot R^{\prime}\right)=0$ then $R^{\prime}$ contains the class of a fiber of the morphism $\operatorname{Exc}\left(R_{1}\right) \simeq \mathbb{P}^{1} \times \mathbb{P}^{2} \rightarrow \mathbb{P}^{2}$. This implies that $R^{\prime}=R_{0}$, which leads to a contradiction. Thus $l\left(R^{\prime}\right)=1$ by Theorem 3.6 (2) and Remark 3.7 (2). Since $s(X) \geq 4$, there exist distinct extremal rays $R_{2}, R_{3}$ apart from $R_{1}$ such that $R_{2}, R_{3}$ are of type (C). We note that $\rho_{X}=4$ by Corollary 2.6. Let $\varphi: X \rightarrow Y$ be the contraction morphism associated to $R_{0}$ and set $D_{i}:=\varphi\left(\operatorname{Exc}\left(R_{i}\right)\right)$ for $1 \leq i \leq 3$. Since $\operatorname{Exc}\left(R_{i}\right)=\varphi^{*} D_{i}, D_{i} \cap D_{j}=\emptyset$ for $1 \leq i<j \leq 3$. By Theorem 3.6 (3), for any $1 \leq i \leq 3$, there exists a contraction morphism $\psi_{i}: Y \rightarrow Z_{i}$ associated to an extremal ray $R_{Z}^{i} \subset \mathrm{NE}(Y)$ such that $\psi_{i}\left(D_{i}\right)$ is a point. Since $\rho_{Y}=3$, each ray $R_{Z}^{i}$ is divisorial by Proposition 2.4 (11). However, this contradicts to Corollary 2.6 (1).

Therefore, we can assume that any extremal ray $R$ with $l(R) \geq 2$ is of fiber type. Since $s(X) \geq 4$, there exist distinct extremal rays $R_{1}, \ldots, R_{m}$ of fiber

type such that $\sum_{i=1}^{m}\left(l\left(R_{i}\right)-1\right) \geq 4$. By Theorem 2.11, $\sum_{i=1}^{m}\left(l\left(R_{i}\right)-1\right)=4$ and $X \simeq \prod_{i=1}^{m} \mathbb{P}^{l\left(R_{i}\right)-1}$.

As a consequence, we have completed the proof of Theorem 1.4 (iii).

\section{References}

[ACO04] M. Andreatta, E. Chierici, and G. Occhetta, Generalized Mukai conjecture for special Fano varieties, Cent. Eur. J. Math. 2 (2004), no. 2, 272-293.

[AO02] M. Andreatta and G. Occhetta, Special rays in the Mori cone of a projective variety, Nagoya Math. J. 168 (2002), 127-137. 
[Ara06] C. Araujo, Rational curves of minimal degree and characterizations of projective spaces, Math. Ann. 335 (2006), no. 4, 937-951.

[BCDD03] L. Bonavero, C. Casagrande, O. Debarre and S. Druel, Sur une conjecture de Mukai. Comment. Math. Helv. 78 (2003), no. 3, 616-626.

[BCW02] L. Bonavero, F. Campana, and J. A. Wiśniewski, Variétés projectives complexes dont l'éclatée en un point est de Fano, C. R. Math. Acad. Sci. Paris 334 (2002), no. 6, 463-468.

[Cas06] C. Casagrande, The number of vertices of a Fano polytope, Ann. Inst. Fourier (Grenoble) 56 (2006), no. 1, 121-130.

[Cas08] C. Casagrande, Quasi-elementary contractions of Fano manifolds, Compos. Math. 144 (2008), no. 6, 1429-1460.

[Cas09] C. Casagrande, On Fano manifolds with a birational contraction sending a divisor to a curve, Michigan Math. J. 58 (2009), no. 3, 783-805.

[CMSB02] K. Cho, Y. Miyaoka and N. I. Shepherd-Barron, Characterizations of projective spaces and applications to complex symplectic manifolds, Higher dimensional birational geometry (Kyoto, 1997), 1-88, Adv. Stud. Pure Math. 35, Math. Soc. Japan, Tokyo, 2002.

[Har77] R. Hartshorne, Algebraic geometry, Graduate Texts in Mathematics, No. 52. Springer-Verlag, New York-Heigelberg, 1977.

[Keb02] S. Kebekus, Families of singular rational curves, J. Algebraic Geom. 11 (2002), no. 2, 245-256

[KM98] J. Kollár and S. Mori, Birational geometry of algebraic varieties, Cambridge Tracts in Math, vol. 134, Cambridge University Press, Cambridge, 1998.

[Kol96] J. Kollár, Rational curves on algebraic varieties, Ergebnisse der Mathematik und ihrer Grenzgebiete, vol. 32, Springer-Verlag, 1996.

[MM81] S. Mori and S. Mukai, Classification of Fano 3-folds with $B_{2} \geq 2$, Manuscripta Math. 36 (1981), no. 2, 147-162. Erratum: 110 (2003), no. 3,407 .

[Mor82] S. Mori, Threefolds whose canonical bundles are not numerically effective, Ann. of Math. (2) 116 (1982), no. 1, 133-176.

[Muk88] S. Mukai, Problems on characterization of the complex projective space, Birational Geometry of Algebraic Varieties, Open Problems, Proceedings of the 23rd Symposium of the Taniguchi Foundation at Katata, Japan, 1988, pp. $57-60$.

[NO10] C. Novelli and G. Occhetta, Rational curves and bounds on the Picard number of Fano manifolds, Geom. Dedicata 147 (2010), 207-217. 
[Occ06] G. Occhetta, A characterization of products of projective spaces, Canad. Math. Bull. 49 (2006), no. 2, 270-280.

[Tsu10a] T. Tsukioka, Fano manifolds obtained by blowing up along curves with maximal Picard number, Manuscripta Math. 132 (2010), no. 1-2, 247-255.

[Tsu10b] T. Tsukioka, A remark on Fano 4-folds having (3,1)-type extremal contractions, Math. Ann. 348 (2010), no. 3, 737-747.

[Tsu10c] T. Tsukioka, Pseudo-index and the length of extremal rays of Fano manifolds (in Japanese), WebProceedings of Mini-Conference on Algebraic Geometry in Saitama University, March 1-2, 2010. See http://www.rimath.saitama-u.ac.jp/lab.jp/fsakai/proc2010e.html/

[Tsu12] T. Tsukioka, On the minimal length of extremal rays for Fano fourfolds, Math. Z. 271 (2012), no. 1-2, 555-564.

[Wiś90] J. A. Wiśniewski, On a conjecture of Mukai, Manuscripta Math. 68 (1990), no. 2, 135-141.

[Wiś91] J. A. Wiśniewski, On contractions of extremal rays of Fano manifolds, J. Reine Angew. Math. 417 (1991), 141-157.

K. Fujita

Research Institute for Mathematical Sciences (RIMS), Kyoto University, Oiwake-cho, Kitashirakawa, Sakyo-ku, Kyoto 606-8502, Japan fujita@kurims.kyoto-u.ac.jp 